necessary, based on clinical history and physical examination, and whenever possible serological tests (ANCA and anti-GBM), to start immunosuppressive therapy to interrupt fatal course of lung and kidney complications.

\section{PO-0792 POST NATAL EVALUATION AND MANAGEMENT OF PRENATAL HYDRONEPHROSIS}

${ }^{1}$ B safaeian, ${ }^{2} S$ Ariannejad. 'Pediatric Nephrology, Taleghani Hospital, Gorgan, Iran; ${ }^{2}$ Pediatric, Taleghani Hospital, Gorgan, Iran

\subsection{6/archdischild-2014-307384.1428}

Background and aims Fetal hydronephrosis is the most common anomaly detected on prenatal ultrasound examination. Several algorithms exist for its management but it remains controversy until now. Here we present our experience in management and outcome of the patients with prenatal hydronephrosis.

Methods In this study 160 known cases of prenatal hydronephrosis were evaluated for detecting underlying aetiology. We carried out renal and bladder sonography and voiding cystourethrography (VCUG) for all patients and DTPA scan in cases with suspicion of obstruction.

Results Of 160 cases of prenatal hydronephrosis 75\% were boys and $25 \%$ were girls. The mean duration of postnatal follow up was 21.5 months (3 months to 36 months). The most common detected abnormalities were ureteropelvic junction obstruction (UPJO) 90 patients, vesicoureteral reflux (VUR) 46 patients, posterior urethral valve (PUV) 15 patients respectively. Nine patients had neurogenic bladder. Prenatal hydronephrosis were unilateral in $83.2 \%$ (133 cases). In these cases $57 \%$ were on the left side . The grade of reflux was severe in 20 renal units $(36.4 \%)$, moderate in 24 renal units (43.6\%), and mild in 11 renal units (20\%). During the follow up period 50 cases (31.25\%) resolved spontaneously. 34 cases $(21.25 \%)$ underwent surgery and 76 $(47.5 \%)$ are still under medical treatment.

Conclusions It is highly suggested to do standard VCUG in all boys with prenatal hydronephsis to detect PUV and neurogenic bladder. Additionally, renal sonography in all cases of congenital hydronephrosis and DTPA in cases with indication is recommended.

\section{PO-0793 STUDY ON THE DETERMINING FACTORS FAVOURING URINARY TRACT INFECTIONS IN CHILDREN}

${ }^{1} \mathrm{C}$ Singer, ${ }^{1} \mathrm{P}$ Stancu, ${ }^{1} \mathrm{~A}$ Morosanu, ${ }^{2} \mathrm{G}$ Oancea, ${ }^{2} \mathrm{C}$ Cristea, ${ }^{2} \mathrm{~A}$ Conea, ${ }^{2} \mathrm{P}$ Popescu, ${ }^{2} \mathrm{~B}$ Dumitru. ${ }^{1} /$ Ind Pediatric Clinic, University of Medicine and Pharmacy of Craiova, Craiova, Romania; ${ }^{2}$ IInd Pediatric Clinic, Universitary Emergency Clinical Hospital, Craiova, Romania

\subsection{6/archdischild-2014-307384.1429}

Background and aims The study of the factors that are in favour and determine the urinary tract infections (UTI) in children hospitalised in Clinic II Paediatrics, SCJU Craiova, between 1.01.2012-31.12.2013.

Material and method 213 children: 48 (22, 5\%) infants, 25 (11.7\%) 1-3 years, $31(14.6 \%) 3-6$ years, and $109(51.2 \%)>6$ years

Results UTI accounted for $4.4 \%$ of all hospital admissions.

Distribution by sex (M/F): Infant $33 / 15,1-3$ years $17 / 8,3-6$ years 14/17, > 6 years 27/82; medium of origin Urban/Rural: Infant $26 / 22$, $1-3$ years $11 / 14,3-6$ years $14 / 17$, > 6 years 42/67.
Favouring factors - Urinary tract abnormalities: phimosis 13 cases, hydronephrosis 12, 11 with kidney stones, vesicoureteral reflux 5 children, and pielocaliceal duplication in 5 children, 4 with hypospadias, congenital kidney in 2; vulvovaginitis in 9; oxiuriaza in 8 ; constipation in 7 ; poor hygiene at 15 .

Bacterial determinant factors: E Coli in 118, Proteus in 28, 25 with Klebsiella, Enterobacter in 13 Pseudomonas aeruginosa in 10; viral causes in 19 cases (acute viral haemorrhagic cystitis). Conclusion 1. UTI were more common in males in infants and children :1-3 years and as for the females in children between $3-6$ years and $>6$ years;

2. E coli was the determining factor in over $50 \%$ of the cases.

3. Urinary tract anomalies favoured about $1 / 4$ of the cases.

\section{PO-0794 NEW TUBULAR INJURY MARKERS IN CHILDREN WITH SOLITARY FUNCTIONING KIDNEY}

K Taranta-Janusz, A Wasilewska, M Dmochowska, M Pszczólkowska, L Janusz. Deapartment of Pediatrics and Nephrology, Medical University of Bialystok, Bialystok, Poland

\subsection{6/archdischild-2014-307384.1430}

The present study aimed to assess whether urinary profiles of the following lysosomal exoglycosidases: $\mathrm{N}$-acethyl- $\beta$-hexosaminidase (HEX), its isoenzymes A (HEX A) and B (HEX B), $\alpha-$ fucosidase (FUC), $\beta$-galactosidase (GAL), $\alpha-$ mannosidase (MAN), and $\beta$ - glucuronidase (GLU) are useful biomarkers of tubular dysfunction in children with solitary functioning kidney (SFK).

Methods We measured HEX, its isoenzymes HEX A, HEX B and FUC, GAL, MAN, GLU urinary activity in 52 patients with SFK. Patients were subdivided in two groups: congenital SFK (cSFK) - unilateral renal agenesis and acquired SFK (aSFK) - unilateral nephrectomy. The reference group (RG) contained 60 healthy children sex and age matched.

Results Urinary activity of all exoglycosidases in SFK was significantly higher than in RG ( $\mathrm{p}<0.05)$. There was no difference in exoglycosidases activity between cSFK and aSFK ( $p>0.05)$. HEX, its isoenzymes HEX A, HEX B negatively correlated with eGFR, and all estimated parameters correlated positively with albumin/ creatinine ratio $(\mathrm{p}<0.001)$.

Conclusion Urinary activity of HEX, its isoenzymes HEX A, HEX B, FUC, GAL, MAN, and GLU is elevated in children with SFK. Long-term follow-up studies in larger groups of children with SFK may help us better understand their clinical significance.

\section{P0-0795 URINARY TRACT INFECTIONS IN CHILDREN AND ROLE OF PAEDIATRICIAN IN THEIR EARLY DETECTION AND TREATMENT}

II Timovski, ${ }^{1} \mathrm{~V}$ Angelovska, ${ }^{2} \mathrm{M}$ Timovska. ${ }^{1}$ Pediatrician, PHI D-R Angelovska and D-R Timovski, Skopje, Macedonia; ${ }^{2}$ Analytics and Research, PRD, Skopje, Macedonia

\subsection{6/archdischild-2014-307384.1431}

Introduction Urinary infections are common problem in paediatric practice. Every vague febrile condition should be considered as a possible urinary infection.

Objectives Role of paediatrician in early detection and treatment in urinary infection 